\title{
Estudio de la Desodorización de Aceite de Soja por Simulación
}

\author{
Marcelo N. Yerien ${ }^{(1)}$, Carlos A. Parodi ${ }^{(1)}$ y Enrique A. Campanella ${ }^{(2,3)}$ \\ (1) Facultad de Ingeniería Química (UNL), Santiago del Estero 2654, (3000) Santa Fe-Argentina \\ (2) INTEC (UNL-CONICET), Güemes 3450, (3000) Santa Fe-Argentina \\ (3) Facultad de Ingeniería y Ciencias Hídricas (UNL), Ruta Nac. Nº168, Km 472.4, \\ (3000) Santa Fe-Argentina (e-mail: tquique@santafe-conicet.gov.ar)
}

Recibido Sept. 01, 2009; Aceptado Oct. 13, 2009; Versión Final recibida Dic. 28, 2009

\section{Resumen}

Se presentan resultados de la simulación de la operación de desodorización de aceites vegetales. Para efectos de simulación, el aceite de soja se modeló como una mezcla multicomponente de cinco compuestos: triglicérido, ácido graso, esterol, tocoferol y escualeno. Se estudiaron las condiciones de operación de flujo cruzado y flujo en contracorriente. Se varió la temperatura, presión y tiempo de contacto, se estudió cantidad de tocoferoles, esteroles y escualeno en el destilado y la presencia de compuestos trans en el aceite. Los resultados obtenidos estuvieron de acuerdo con resultados experimentales, e indicaron que el flujo en contracorriente es más conveniente desde el punto de vista de la calidad del destilado y del aceite, así como del consumo de vapor.

Palabras claves: simulación de procesos, desodorización, aceite de soja, modelado

\section{Study of Soybean Oil Deodorization using Simulation}

\begin{abstract}
This work presents simulation results of a vegetable oil deodorization process. For simulation purposes, the soybean oil was modelled as a five component mixture: triglyceride, fatty acid, sterol, tocopherol and squalene. Two types of flow, cross and countercurrent, were studied. The process temperature, pressure and contact time were changed, the amount of tocopherol, sterol and squalene in the distillate and the presence of trans compounds in the vegetable oil were analyzed. The results were in agreement with experiment and indicate that countercurrent flow is more convenient from the point of view of quality of the distillate and of the oil, as well as of steam consumption.
\end{abstract}

Keywords: process simulation, deodorization, soybean oil, modeling 


\section{INTRODUCCION}

La desodorización es una etapa en el proceso de refinación de los aceites vegetales. Si la refinación es física la desodorización es un proceso de agotamiento con vapor (Balchen et al., 1999). El agotamiento con vapor implica que junto a la operación física de extracción con vapor ocurren reacciones químicas que pueden afectar la calidad del aceite (Maza et al., 1992). Las condiciones de operación del agotamiento también afectan el destilado de la desodorización (Verleyen et al, 2001) que contiene valiosos compuestos cuya recuperación tiene gran potencial económico (Hirota et al., 2003; Moreira y Baltanás, 2001; Winters, 1994). Recientemente se ha realizado una importante contribución (Ceriani y Meirelles, 2004a) para describir el equilibrio líquido-vapor de sistemas relacionados con los ácidos grasos necesarios en la simulación de la operación de agotamiento. Por otro lado, en la literatura se pueden encontrar trabajos que han simulado la desodorización batch (Ceriani y Meirelles, 2004b; Balchen et al., 1999), la continua (Ceriani y Meirelles, 2004c, 2006) y la formación de ácidos grasos trans (Ceriani et al., 2008). En este trabajo se presentan resultados de la simulación del agotamiento continuo con vapor de un aceite de soja.

\section{BASE DE CÁLCULO DE LAS SIMULACIONES}

La desodorización de los aceites vegetales es una refinación física del aceite para obtener un producto con sabor y olor aceptables. Existen varias alternativas para el proceso, en este trabajo sólo se consideró el agotamiento con vapor en dos configuraciones, flujo cruzado y contracorriente. Todas las simulaciones fueron realizadas a partir de un caso base industrial (Ceriani y Meirelles, 2004c). La Tabla 1 presenta los datos básicos de esa desodorización industrial.

Tabla 1: Datos de una desodorización industrial de aceite de soja

\begin{tabular}{lc}
\hline Parámetro & Flujo cruzado \\
\hline Flujo de aceite, $[\mathrm{kg} / \mathrm{hr}]$ & 4425 \\
Flujo total de vapor (1.3\% del flujo de aceite), $[\mathrm{kg} / \mathrm{hr}]$ & 57 (19 por separador) \\
Número de etapas (separadores) & 3 \\
Temperatura, $\left[{ }^{\circ} \mathrm{C}\right]$ & 242 \\
Presión, $[\mathrm{mmHg}]$ & 1 \\
Caída de presión & Despreciable \\
\hline
\end{tabular}

El aceite de soja fue reducido a una mezcla de cinco componentes representantes de las especies químicas de interés en el proceso. Un triglicérido que representa a la mezcla de triglicéridos (TAG), diglicéridos (DAG) y monoglicéridos (MAG), un ácido graso que representa a la mezcla de ácidos grasos libres del aceite vegetal, $\beta$-sitosterol que representa a los esteroles, $\delta$-tocoferol que representa a los tocoferoles y el escualeno que representa al resto de los componentes minoritarios. El triglicérido es OLiLi (oleico, linoleico, linoleico) seleccionado con el procedimiento recomendado por Campanella et al. (2009). El ácido graso libre es el ácido linoleico, el más significativo de la mezcla. Las propiedades físicas de los compuestos, presión de vapor inclusive, se obtuvieron de la literatura. Los datos faltantes se estimaron con métodos tradicionales (Poling et al., 2001). El equilibrio líquido-vapor se trata de la siguiente manera:

$y_{i} \phi_{i} P=P_{i}^{0} \gamma_{i} x_{i} C P$

donde $y_{i}$ e $x_{i}$ son las fracciones molares de vapor y líquido respectivamente, $\phi_{i}$ el coeficiente de fugacidad, $P$ la presión de trabajo, $P_{i}^{0}$ la presión de vapor, $\gamma_{i}$ el coeficiente de actividad y $C P$ la corrección de Poynting. En este trabajo se despreció el efecto de $\phi_{i}$ y de CP. Los coeficientes de actividad se calcularon con UNIQUAC y los parámetros de interacción se obtuvieron con UNIFAC. 
Se estudiaron dos configuraciones. La desodorización en flujo cruzado, que consiste en una serie de platos con el aceite alimentado en el tope y con el vapor que se suministra en cada plato en forma independiente. La Fig. 1 muestra esquemáticamente la configuración en flujo cruzado y el diagrama de flujo de la simulación. La desodorización en contracorriente es la tradicional torre de agotamiento con vapor directo. La Fig. 2 muestra esquemáticamente la configuración en contracorriente y el diagrama de flujo de la simulación. Los esquemas de simulación permiten el arrastre por vapor del aceite vegetal con los mezcladores y divisores de corriente. Además, es posible incorporar una eficiencia de Murphree en cada etapa de la torre desodorizadora. Se realizaron simulaciones con y sin arrastre, y con y sin eficiencia. Las simulaciones se realizaron con dos simuladores comerciales equivalentes (HYSYS y UniSim Design).

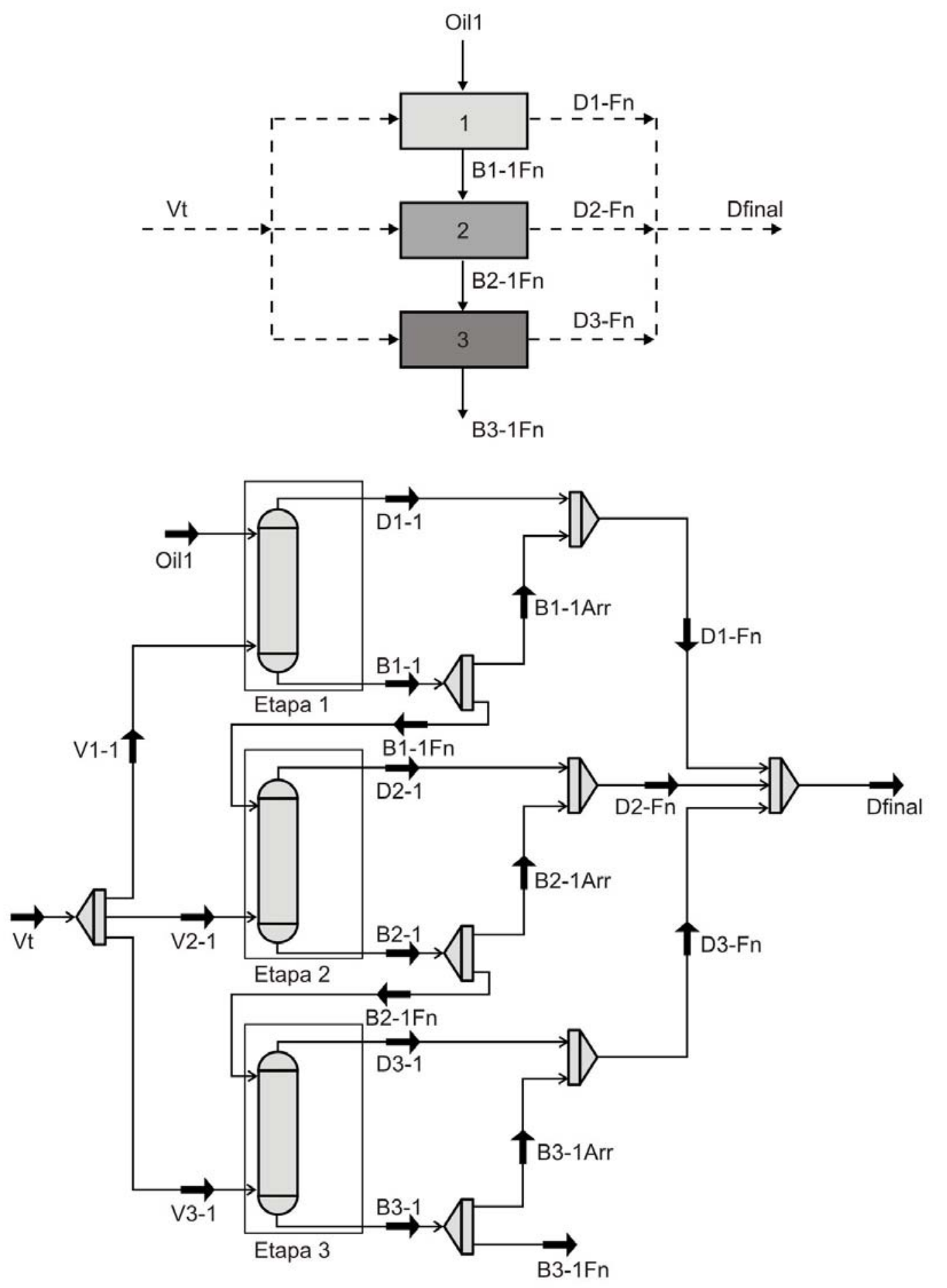

Fig. 1: Diagrama de flujo para un diseño de flujo cruzado y su diagrama de simulación 

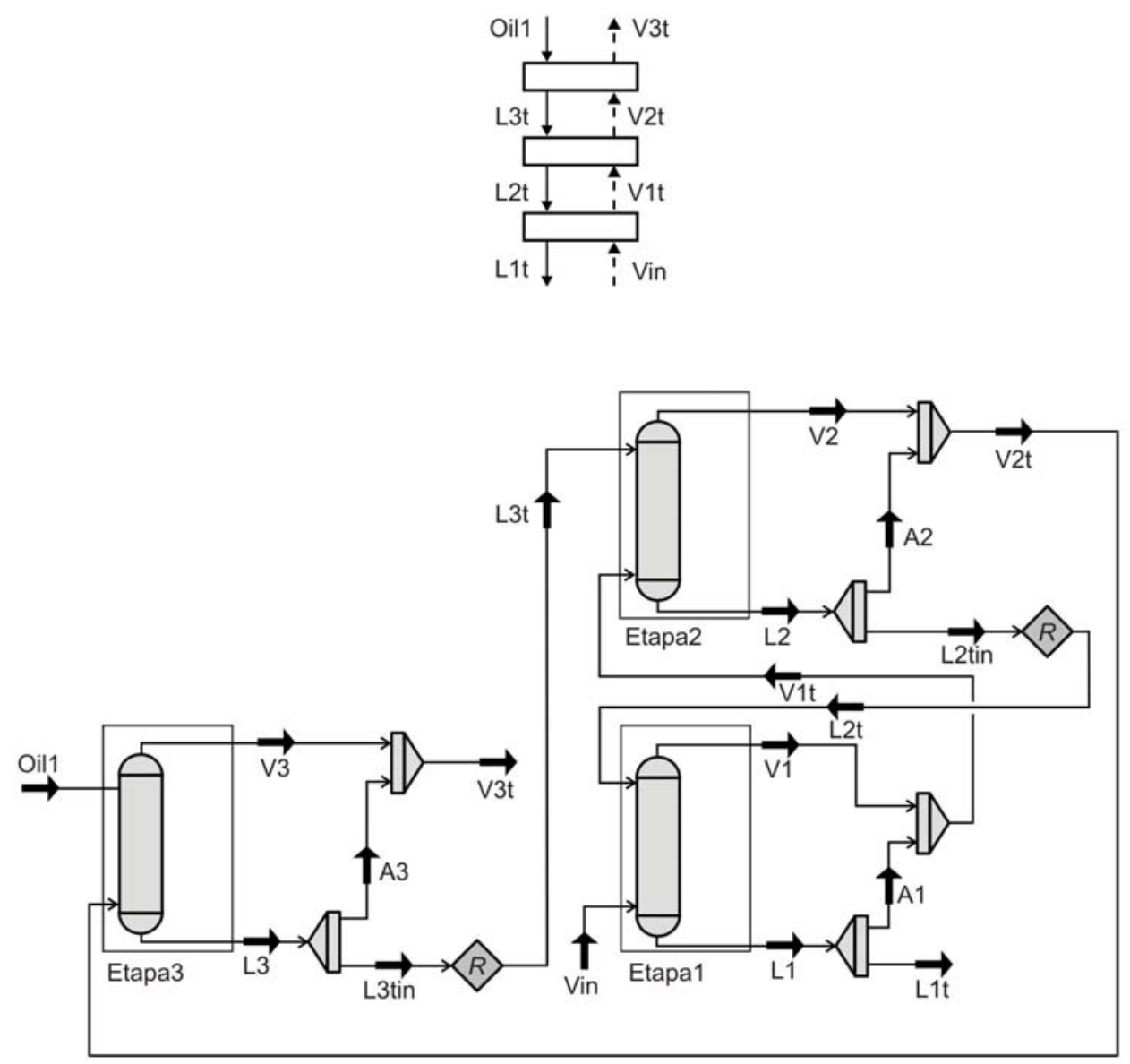

Fig. 2: Diagrama de flujo para un diseño de flujo en contracorriente y su diagrama de simulación

\section{RESULTADOS Y DISCUSIÓN}

\section{Simulación base}

Las primeras simulaciones de la desodorización del aceite de soja se realizaron con los datos correspondientes a la desodorización industrial presentados en la Tabla 1. La composición del aceite en base a los cinco componentes representativos es la que muestra la Tabla 2. Se exploraron diferentes condiciones, se cambió la presión de operación en el rango de 1 a $6 \mathrm{mmHg}$ y el caudal de vapor en el rango de 1 a $2 \%$ del caudal de aceite. Se consideró flujo cruzado y en contracorriente para una columna de 3 platos teóricos. Para el caso en contracorriente se simularon columnas de 5 y 10 platos. Finalmente se considera a la operación con 100 y $50 \%$ de eficiencia. Para incluir el arrastre del aceite por el vapor se agregó en el diagrama de flujo de la simulación un divisor de corriente que agrega líquido al vapor que abandona la etapa. En la mayoría de los desodorizadores industriales la pérdida de aceite por arrastre es del $0.2 \%$. En las simulaciones realizadas se presenta una situación más extrema adoptando un valor para el parámetro del divisor de corriente de 0.023, lo que origina una pérdida de aceite mucho mayor.

Los principales resultados se muestran en las Tablas 3, 4 y 5. Las simulaciones de las tablas fueron realizadas con 3 platos teóricos y una eficiencia de 0.5 . La concentración de los compuestos en las tablas se expresa en gramos por 100 gramos de destilado libre de agua. La retención de los productos valiosos (escualeno, tocoferol, sitosterol) es el porcentaje que queda en los destilados, y la acidez del aceite es el porcentaje de ácido linoleico. Las tablas permiten apreciar, para sus respectivas disposiciones de flujos, la influencia de las condiciones de proceso sobre la calidad del destilado y del aceite en el fondo del equipo. Se aprecian composiciones del destilado como función de la temperatura, obtenidas con $1.3 \%$ de vapor de extracción. 
Tabla 2: Composición del aceite de soja utilizado en las simulaciones

\begin{tabular}{|l|c|}
\hline Componente & Fracción másica (\%) \\
\hline TAG (oleico, linoleico, linoleico), incluye DAG y MAG & 99.450 \\
\hline FFA (linoleico) & 0.070 \\
\hline Escualeno & 0.014 \\
\hline$\beta$-Sitosterol & 0.330 \\
\hline$\delta$-Tocoferol & 0.136 \\
\hline Peso molecular, [g/gmol] & 864.61 \\
\hline
\end{tabular}

Tabla 3: Composición del Destilado para diferentes temperaturas y P = $1 \mathrm{mmHg}$

\begin{tabular}{|l|c|c|c|c|c|c|}
\cline { 2 - 7 } \multicolumn{1}{c|}{} & \multicolumn{3}{c|}{ Flujo en Corriente cruzada } & \multicolumn{3}{c|}{ Flujo en Contracorriente } \\
\cline { 2 - 7 } \multicolumn{1}{c|}{} & $24{ }^{\circ} \mathrm{C}$ & $250^{\circ} \mathrm{C}$ & $258{ }^{\circ} \mathrm{C}$ & $242{ }^{\circ} \mathrm{C}$ & $250{ }^{\circ} \mathrm{C}$ & $258^{\circ} \mathrm{C}$ \\
\hline Destilado, $[\mathrm{kg} / \mathrm{h}]$ & 0.48 & 0.57 & 0.69 & 9.02 & 11.19 & 13.89 \\
\hline OLiLi & $4.78 \mathrm{E}-07$ & $1.34 \mathrm{E}-06$ & $3.64 \mathrm{E}-06$ & $1.07 \mathrm{E}-06$ & $2.86 \mathrm{E}-06$ & $7.38 \mathrm{E}-06$ \\
\hline Ac. Linoleico & 46.932 & 40.129 & 34.298 & 34.849 & 27.682 & 22.304 \\
\hline Escualeno & 5.912 & 5.212 & 4.544 & 7.113 & 5.502 & 4.447 \\
\hline$\%$ Ret.Escualeno & 91.65 & 94.81 & 96.87 & 98.56 & 99.36 & 99.69 \\
\hline$\beta$-Sitosterol & 27.464 & 32.433 & 37.080 & 33.167 & 38.911 & 44.113 \\
\hline$\%$ Ret. $\beta$-Sitosterol & 18.24 & 25.27 & 33.86 & 20.49 & 29.81 & 41.95 \\
\hline$\delta$-Tocoferol & 19.692 & 22.226 & 24.078 & 24.871 & 27.905 & 29.136 \\
\hline$\%$ Ret. $\delta$-Tocoferol & 30.81 & 40.81 & 51.81 & 37.31 & 51.88 & 67.23 \\
\hline Acidez aceite, $[\%]$ & 0.00045 & 0.00021 & 0.00010 & $3.12 \mathrm{E}-05$ & $1.21 \mathrm{E}-05$ & $5.14 \mathrm{E}-06$ \\
\hline
\end{tabular}

Tabla 4: Composición del Destilado para diferentes temperaturas y P $=2.775 \mathrm{mmHg}$

\begin{tabular}{|c|c|c|c|c|c|c|c|c|}
\hline & \multicolumn{4}{|c|}{ Flujo en Corriente cruzada } & \multicolumn{4}{|c|}{ Flujo en Contracorriente } \\
\hline & \multirow{2}{*}{$242^{\circ} \mathrm{C}$} & \multicolumn{2}{|c|}{$250^{\circ} \mathrm{C}$} & \multirow{2}{*}{$258^{\circ} \mathrm{C}$} & \multirow{2}{*}{$242^{\circ} \mathrm{C}$} & \multicolumn{2}{|c|}{$250^{\circ} \mathrm{C}$} & \multirow{2}{*}{$258^{\circ} \mathrm{C}$} \\
\hline & & C/Arrastre & S/Arrastre & & & C/Arrastre & S/Arrastre & \\
\hline Destilado, [kg/h] & 0.31 & 36.50 & 0.36 & 0.42 & 5.52 & 110.56 & 6.48 & 7.74 \\
\hline OLiLi & 2.55E-07 & 94.06 & 7.34E-07 & 2.03E-06 & 6.28E-07 & 93.703 & $1.78 \mathrm{E}-06$ & 4.77E-06 \\
\hline Ac. Linoleico & 65.548 & 2.991 & 58.905 & 52.050 & 55.643 & 2.795 & 47.710 & 39.944 \\
\hline Escualeno & 6.475 & 0.328 & 6.304 & 5.956 & 9.505 & 0.517 & 8.792 & 7.626 \\
\hline \% Ret.Escualeno & 68.08 & 78.02 & 75.80 & 82.31 & 84.68 & 92.26 & 91.92 & 95.33 \\
\hline$\beta$-Sitosterol & 15.842 & 1.622 & 19.892 & 24.298 & 19.645 & 1.752 & 24.621 & 29.843 \\
\hline$\%$ Ret. $\beta$-Sitosterol & 7.14 & 16.51 & 10.25 & 14.39 & 7.43 & 13.27 & 10.92 & 15.82 \\
\hline$\delta$-Tocoferol & 12.135 & 0.999 & 14.899 & 17.696 & 15.207 & 1.233 & 18.877 & 22.586 \\
\hline$\%$ Ret. $\delta$-Tocoferol & 12.88 & 23.95 & 18.08 & 24.68 & 13.95 & 22.65 & 20.32 & 29.06 \\
\hline Acidez aceite, [\%] & 0.00412 & 0.00219 & 0.00229 & 0.00124 & 5.08E-04 & 2.34E-04 & 2.34E-04 & $1.08 \mathrm{E}-04$ \\
\hline
\end{tabular}

Como era de esperar el caudal de destilado se incrementa con la temperatura y decrece con el aumento de presión del sistema. La simulación incluye el efecto de arrastre del aceite con vapor, que llevó a un incremento considerable del OLiLi (desde aproximadamente 1.E-07 a $94 \%$ para ambas disposiciones de flujo), aclarando que todos los procesos reales tienen arrastre de aceite con vapor. Dependiendo de las condiciones de temperatura y presión, para corriente cruzada el destilado presentó importantes cantidades de tocoferol y sitosterol. Para temperaturas superiores a $250{ }^{\circ} \mathrm{C}$, la 
simulación mostró, dependiendo de la presión del sistema (conforme la presión disminuye aumenta la retención), que es posible recuperar entre 12.57 y $51.81 \%$ de tocoferol y entre 7.00 y $33.86 \%$ de sitosterol. A temperatura de $242^{\circ} \mathrm{C}$ la retención disminuye en aproximadamente un $52 \%$ para ambos compuestos independientemente de la presión del sistema. Cuando el proceso es llevado a cabo en contracorriente se produce un aumento considerable en el caudal de destilado, a iguales condiciones de operación, que llega a alcanzar valores de alrededor de 20 veces el obtenido en corriente cruzada, con apreciable enriquecimiento en la composición de tocoferoles, esteroles y escualenos. Por lo tanto es conveniente utilizar la disposición en contracorriente si el destilado es importante en la economía de la planta.

Tabla 5: Composición del Destilado para diferentes temperaturas y $\mathrm{P}=6 \mathrm{mmHg}$

\begin{tabular}{|c|c|c|c|c|c|c|}
\hline & \multicolumn{3}{|c|}{ Flujo en Corriente cruzada } & \multicolumn{3}{|c|}{ Flujo en Contracorriente } \\
\hline & $242^{\circ} \mathrm{C}$ & $250^{\circ} \mathrm{C}$ & $258^{\circ} \mathrm{C}$ & $242^{\circ} \mathrm{C}$ & $250^{\circ} \mathrm{C}$ & $258^{\circ} \mathrm{C}$ \\
\hline Destilado, $[\mathrm{kg} / \mathrm{h}]$ & 0.22 & 0.26 & 0.30 & 4.17 & 4.73 & 5.44 \\
\hline OLiLi & $1.60 \mathrm{E}-07$ & 4.58E-07 & 1.27E-06 & 3.84E-07 & $1.13 E-06$ & $3.14 \mathrm{E}-06$ \\
\hline Ac. Linoleico & 76.125 & 71.392 & 65.877 & 70.241 & 63.567 & 56.392 \\
\hline Escualeno & 5.692 & 5.843 & 5.904 & 8.424 & 8.792 & 8.851 \\
\hline \% Ret.Escualeno & 44.10 & 52.23 & 60.32 & 56.74 & 67.12 & 77.75 \\
\hline$\beta$-Sitosterol & 10.197 & 12.841 & 16.031 & 12.014 & 15.600 & 19.670 \\
\hline$\%$ Ret. $\beta$-Sitosterol & 3.38 & 4.92 & 7.02 & 3.43 & 5.05 & 7.33 \\
\hline$\delta$-Tocoferol & 7.987 & 9.925 & 12.188 & 9.321 & 12.040 & 15.087 \\
\hline$\%$ Ret. $\delta$-Tocoferol & 6.25 & 8.95 & 12.57 & 6.46 & 9.46 & 13.64 \\
\hline Acidez aceite, [\%] & 0.01362 & 0.00900 & 0.00567 & 4.07E-03 & $1.95 \mathrm{E}-03$ & 9.05E-04 \\
\hline
\end{tabular}

\section{Otras simulaciones}

Primero se trabajó la respuesta del simulador comparando datos experimentales proporcionados en el trabajo de Maza et al. (1992), donde se estudia el proceso continuo. El objetivo del trabajo de Maza fue evaluar la calidad del aceite cambiando condiciones del proceso. La Fig. 3 compara los resultados obtenidos por simulación con los resultados experimentales para la retención de tocoferol. Los dos gráficos muestran idéntica tendencia y valores al cambiar temperatura y presión de operación.
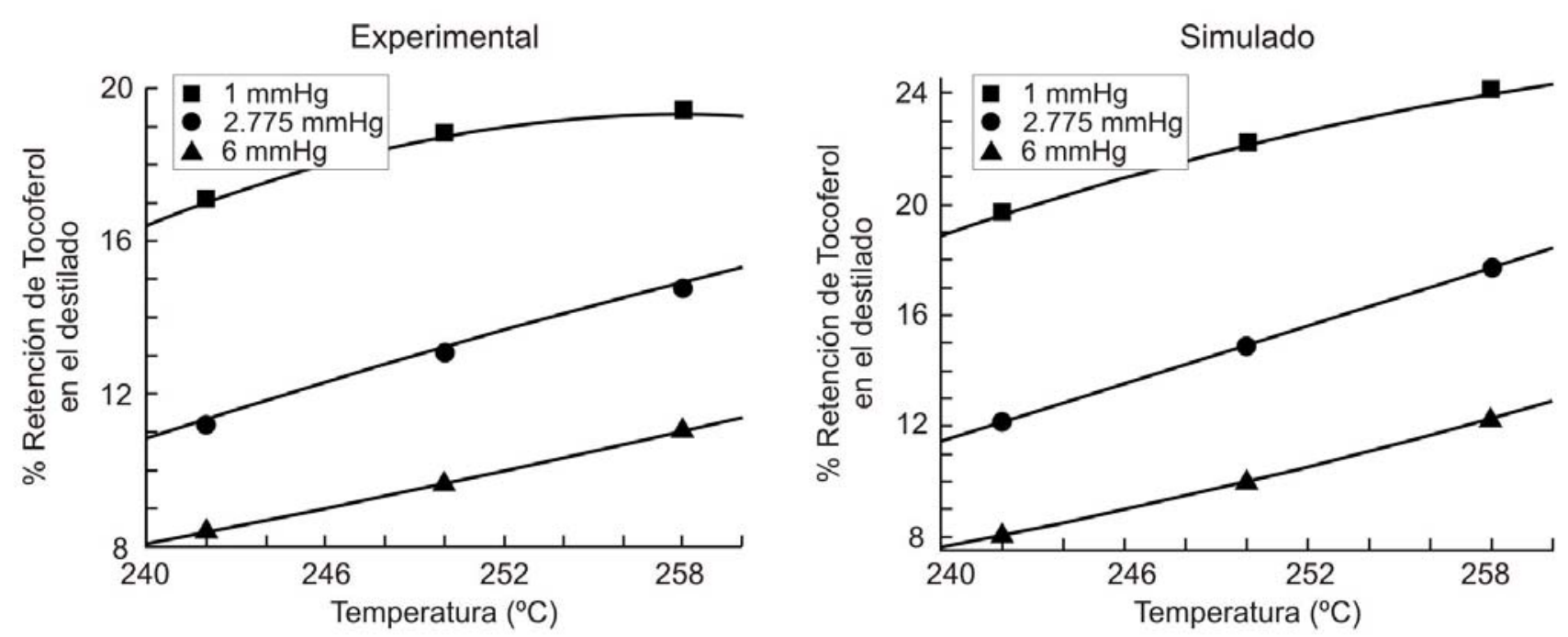

Fig. 3: Retención de tocoferol en experimentos y en simulación. 
Luego se analizó la respuesta del simulador para varias condiciones de operación examinando la calidad del aceite con dos parámetros: la isomerización cis-trans y la retención de tocoferol, sitosterol y escualeno. Para tener en cuenta la isomerización se agregó un nuevo componente (OLiLi trans), la reacción cis-trans en cada etapa del desodorizador (Yerien et al., 2007) y se realizó un diseño factorial en dos niveles para la temperatura $\left(220\right.$ y $\left.260^{\circ} \mathrm{C}\right)$, la presión $(1$ y $6 \mathrm{mmHg})$, el tiempo $(90$ y 240 minutos), y el caudal de vapor (1 y $2 \%$ ). El análisis tuvo dos etapas: la primera para seleccionar las variables más significativas y la segunda para obtener un mapa de respuestas de esas variables.

Para ambas disposiciones de flujo la primera etapa del análisis indica que las variables más significativas son la temperatura y el tiempo. En cambio para las retenciones de tocoferol, sitosterol y escualeno las variables significativas son temperatura, presión y caudal de vapor.

En la segunda etapa se obtuvieron las ecuaciones (2) y (3) para el mapa de respuestas de las variables más significativas. La ecuación (2) representa el porcentaje de isómero trans formado en función de temperatura (T) y tiempo (t). La ecuación (3) representa el porcentaje de retención de A, donde A simboliza escualeno, sitosterol o tocoferol, en función de temperatura $(T)$, presión $(P)$ y caudal de vapor (\% Vap).

$\%$ OLiLi trans $=\alpha_{0}+\alpha_{1}{ }^{*} T+\alpha_{2}{ }^{*} t+\alpha_{3}{ }^{*} T * t+\alpha_{4}{ }^{*} T^{2}+\alpha_{5}{ }^{*} t^{2}$

$$
\begin{aligned}
\% A= & \alpha_{0}+\alpha_{1}{ }^{*} T+\alpha_{2}{ }^{*} P+\alpha_{3} * \% \operatorname{Vap}+\alpha_{4}{ }^{*} T * P+\alpha_{5}{ }^{*} T * \% \operatorname{Vap}+ \\
& +\alpha_{6}{ }^{*} P * \% \operatorname{Vap}+\alpha_{7}{ }^{*} T^{2}+\alpha_{8}{ }^{*} P^{2}+\alpha_{9}{ }^{*} \% \operatorname{Vap}^{2}
\end{aligned}
$$

La Tabla 6 presenta los coeficientes $\left(\alpha_{i}\right)$ de las ecuaciones (2) y (3). Se puede apreciar que el nivel de confianza $\left(R^{2}\right)$ del modelo cuadrático de las ecuaciones (2) y (3) está entre 96 y $100 \%$. Con la ayuda de las ecuaciones es posible comparar los flujos cruzado y contracorriente. Fijando como objetivo una retención de tocoferol de $85 \%$ y una generación de $1 \%$ para los compuestos trans, a una presión de operación de $3.7 \mathrm{mmHg}$, con un tiempo de retención de 90 minutos, las ecuaciones (2) y (3) indican que es necesario un caudal de vapor de $1.52 \%$ de la alimentación para el flujo cruzado y de $1.35 \%$ de la alimentación para el flujo en contracorriente. Es decir que es necesario un caudal de vapor un $13 \%$ mayor para el flujo cruzado.

Tabla 6: Coeficientes para las ecuaciones del mapa de respuestas

\begin{tabular}{|l|c|c|c|c|c|c|c|c|}
\hline & \multicolumn{4}{|c|}{ Flujo en Corriente cruzada } & \multicolumn{4}{c|}{ Flujo en Contracorriente } \\
\hline Coeficiente & OLiLi trans & \% Esc. & \% Sit. & \% Toc. & OLiLi trans & \% Esc. & \% Sit. & \% Toc. \\
\hline$\alpha_{0}$ & 266.064 & 436.517 & 172.161 & 209.151 & 276.143 & 658.782 & 328.505 & 369.028 \\
\hline$\alpha_{1}$ & -2.153 & -2.788 & 2.739 & 3.233 & -2.231 & -4.948 & 4.180 & 4.782 \\
\hline$\alpha_{2}$ & -0.365 & 39.865 & -22.552 & -26.588 & -0.380 & 65.115 & -31.577 & -35.124 \\
\hline$\alpha_{3}$ & 1.24 E-03 & -52.911 & 37.144 & 43.238 & 1.30 E-03 & -82.506 & 53.346 & 56.310 \\
\hline$\alpha_{4}$ & 4.41 E-03 & -0.090 & 0.124 & 0.158 & 4.56 E-03 & -0.211 & 0.169 & 0.209 \\
\hline$\alpha_{5}$ & 3.37 E-04 & 0.094 & -0.218 & -0.264 & 3.43 E-04 & 0.253 & -0.299 & -0.324 \\
\hline$\alpha_{6}$ & & -1.670 & 1.749 & 2.090 & & -3.259 & 2.470 & 2.796 \\
\hline$\alpha_{7}$ & & 0.004 & -0.007 & -0.008 & & 0.009 & -0.010 & -0.012 \\
\hline$\alpha_{8}$ & & -1.031 & -0.913 & -1.304 & & -0.303 & -1.189 & -1.795 \\
\hline$\alpha_{9}$ & & 6.621 & 0.874 & 1.164 & & 6.014 & 0.663 & 0.180 \\
\hline & & & & & & & & \\
\hline $\mathrm{R}^{2}$ & 0.997 & 0.986 & 0.977 & 0.989 & 0.996 & 0.976 & 0.963 & 0.987 \\
\hline
\end{tabular}




\section{CONCLUSIONES}

Los resultados obtenidos muestran que la simulación puede reproducir resultados experimentales, por lo tanto puede ser utilizada para optimizar el proceso de desodorización de aceites vegetales. La incorporación de la reacción de producción de compuestos trans en cada etapa del desodorizador permitió que el análisis de la calidad del aceite incluya además de la recuperación de tocoferol, sitosterol y escualeno, la presencia de compuestos trans. Los resultados indican que es conveniente utilizar el flujo en contracorriente ya que no solo permite una mayor recuperación de compuestos valiosos sino que también permite un ahorro en el vapor de arrastre.

\section{AGRADECIMIENTOS}

Este trabajo ha sido apoyado económicamente por la Universidad Nacional del Litoral, el CONICET y la ANPCyT.

\section{REFERENCIAS}

Balchen, S., R. Gani y J. Adler-Nissen, Deodorization Principles: Stripping Efficiency in Cross-Flow and Counter-Current Operations, INFORM: 10, 245-262 (1999).

Campanella, A., B.A. Mandagarán y E.A. Campanella, Partitioning of Carboxylic Acid between Oil and Water Phases. Experimental, Correlation, and Prediction, J. Am. Oil Chem. Soc.: 86, 513-519 (2009).

Ceriani, R., A.M. Costa y A.J.A. Meirelles, Optimization of the Physical Refining of Sunflower Oil Concerning the Final Contents of trans-Fatty Acids, Ind. Eng. Chem. Res.: 47, 681-692 (2008).

Ceriani, R. y A.J.A. Meirelles, Simulation of Continuous Physical Refiners for Edible Oil Deacidification, J. Food Eng.: 76, 261-271 (2006).

Ceriani, R. y A.J.A. Meirelles, Simulation of Continuous Deodorizers: Effects on Product Streams, J. Am. Oil Chem. Soc.: 81, 1059-1069 (2004c).

Ceriani, R. y A.J.A. Meirelles, Simulation of Batch Physical Refining and Deodorization Processes, J. Am. Oil Chem. Soc.: 81, 305-312 (2004b).

Ceriani, R. y A.J.A. Meirelles, Predicting Vapor-Liquid Equilibria of Fatty Systems, Fluid Phase Equilib.: 215, 227-236 (2004a).

Hirota, Y. y otros siete autores, Purification of Steryl Esters from Soybean Oil Deodorizer Distillate, J. Am. Oil Chem. Soc.: 80, 341-346 (2003).

HYSYS 3.2, Hyprotech: Calgary, Alberta, Canada (2004).

Maza, A., R.A. Ormsbee y L.R. Strecker, Effects of Deodorization and Steam-Refining Parameters on Finished Oil Quality, J. Am. Oil Chem. Soc.: 69, 1003-1008 (1992).

Moreira, E. y M.A. Baltanás, Recuperación de Tocoferoles y Esteroles desde Destilados de Desodorización y otras Materias Primas. Parte 1, A\&G: 43, 253-259 (2001).

Poling, B.E, J.M. Prausnitz y J.P. O'Connell, The Properties of Gases and Liquids, $5^{\text {th }}$ ed., McGrawHill, New York (2001).

UniSim.Design R380, Honeywell: London, Ontario (2008).

Verleyen, T. y otros cinco autores, Gas Chromatographic Characterization of Vegetable Oil Deodorization Distillate, J. Chromatogr. A: 921, 277-285 (2001).

Winters, R.L., Valor y Utilización de los Destilados del Proceso de Desodorización en el Procesamiento de Aceites Comestibles, A\&G Técnica: Dic., 54-61 (1994).

Yerien, M., C.A. Parodi y E.A. Campanella, Estudio del Agotamiento con Vapor de Aceites Vegetales, $11^{\circ}$ Encuentro de Jóvenes Investigadores de la Universidad Nacional del Litoral - 2do. Encuentro de Jóvenes Investigadores de Universidades de Santa Fe, Santa Fe - Argentina, 10 al 11 de Octubre (2007). 